\title{
ASYMPTOTIC BEHAVIOR OF SOLUTIONS OF NONLINEAR VOLTERRA EQUATIONS
}

\author{
BY R. K. MILLER
}

Communicated by F. John, September 9, 1965

In this note we show how certain known results for delay differential equations can be extended to systems of integral equations of the form

$$
x(t)=f(t)+\int_{0}^{t} a(t-s) g(s, x(s)) d s \quad(t \geqq 0) .
$$

We make the following assumptions:

(A1) $f(t)$ is uniformly continuous and bounded on $0 \leqq t<\infty$,

(A2) $a(t)$ is a square matrix whose entries are $L_{1}(0, \infty)$,

(A3) $g(t, x)$ is continuous in $(t, x)$ for $0 \leqq t<\infty,|x|<\infty$ and $g$ is uniformly almost periodic in $t$ uniformly on compact subsets of $x$ in real $n$-space $R^{n}$, and

(A4) $x(t)$ is a bounded solution of (1) for $0 \leqq t<\infty$.

Let $\Omega$ be the positive limit set of $x(t)$. We refer to [2] for the definitions and properties of almost periodic functions and limit sets. The analog for integral equations of [2, Theorem 1] is

THEOREM 1. If (A1)-(A4) are satisfied, then to each point $z$ in $\Omega$ there corresponds a sequence $t_{m} \rightarrow \infty$ as $m \rightarrow \infty$ and functions $G(t, x)$, $X(t)$ and $F(t)$ such that

(i) limit $_{m \rightarrow \infty}\left|x\left(t+t_{m}\right)-X(t)\right|+\left|f\left(t+t_{m}\right)-F(t)\right|=0$ uniformly on compact subsets of $-\infty<t<\infty$,

(ii) limit lim $_{m} g\left(t+t_{m}, x\right)=G(t, x)$ uniformly for all $t$ and for $x$ on compact sets, and

(iii) on the interval $-\infty<t<\infty, X(t) \in \Omega$ and

$$
X(t)=F(t)+\int_{-\infty}^{t} a(t-s) G(s, X(s)) d s
$$

PROof. As is well known in harmonic analysis the convolution of an $L_{1}$ function with an essentially bounded function yields a uniformly continuous function. Hence $x(t)$ is bounded and uniformly continuous on the interval $0 \leqq t<\infty$.

Given $z$ in $\Omega$ let $\left\{t_{m}\right\}$ be a sequence such that $t_{m} \rightarrow \infty$ and $x\left(t_{m}\right) \rightarrow z$ as $m \rightarrow \infty$. Define $x_{m}(t)=x\left(t+t_{m}\right)$ and $f_{m}(t)=f\left(t+t_{m}\right)$ for $t \geqq-t_{m}$. Since

$$
x_{m}(t)=f_{m}(t)+\int_{-t_{m}}^{t} a(t-s) g\left(s+t_{m}, x_{m}(s)\right) d s,
$$


the proof can now be completed in the same way as the proof of [2, Theorem 1].

We remark that with essentially the same proof one can establish a modified version of Theorem 1 in which the lower limit of integration in equation (1) is $-\infty$. Note also that one could add to the right side of (1) a bounded measurable function $h(t) \rightarrow 0$ as $t \rightarrow \infty$.

Since a bounded continuous function must tend to its positive limit set, Theorem 1 above can sometimes be used to obtain results on the asymptotic behavior of solutions. We shall illustrate the technique with some examples. Consider the scalar equation

$$
x(t)=f(t)-\int_{0}^{t} a(t-s) x(s) d s .
$$

Paley and Wiener [4, pp. 58-63] prove:

Theorem 2 (Paley-Wiener). Suppose $a(t)$ is $L_{1}(0, \infty)$ and $f(t)$ is bounded, measurable and tends to a limit $f_{0}$ as $t \rightarrow \infty$. For each such $f$ the solution of (2.1) is bounded and tends to the limit

$$
x(t) \rightarrow x_{0}=f_{0} /\left(1+\int_{0}^{\infty} a(s) d s\right) \text { as } t \rightarrow \infty
$$

if and only if when $\operatorname{Re}(u) \geqq 0$ one has

$$
\int_{0}^{\infty} a(t) \exp (-u t) d t \neq-1
$$

To this we add

Corollary 1. Let $a(t)$ and $f(t)$ be as in Theorem 2. All bounded solutions of (3) satisfy (4) if and only if (5) holds whenever $\operatorname{Re}(u)=0$.

Under the hypothesis of Corollary 1 some solutions may be unbounded as $t \rightarrow \infty$. If we do have a bounded solution, then Theorem 1 above applies. The limiting system corresponding to (2) is in this case

$$
X(t)=f_{0}-\int_{-\infty}^{t} a(t-s) X(s) d s .
$$

The transformation $Y(t)=X(t)-x_{0}$ gives

$$
Y(t)=-\int_{-\infty}^{t} a(t-s) Y(s) d s \quad(-\infty<t<\infty) .
$$

For this last equation it is known that $Y(t) \equiv 0$ is the only bounded 
solution if and only if the Fourier transform of $a(t)$ is never -1 , cf. $[4$, p. 59 and p. 63].

Levin [1] has proved a nonlinear version of Theorem 2. Consider

$$
x(t)=f(t)-\int_{0}^{t} a(t-s) g(x(s)) d s,
$$

with the following assumptions:

(B1) $f$ is bounded and measurable on $0 \leqq t<x$ and tends to $f_{0}$ as $t \rightarrow \infty$,

(B2) $g(x)$ is $C(-\infty, \infty), g(0)=0$, and $g$ is strictly increasing, and

(B3) $a(t)$ is $C[0, \infty), C^{1}(0, \infty)$ and $L_{1}(0, \infty), a(t) \geqq 0, a^{\prime}(t) \leqq 0$ and $a^{\prime}(t) \not \equiv 0$ on any interval except possibly $a^{\prime \prime}(t) \equiv 0$ for all large $t$.

It is possible to separate the boundedness criterion in Levin's problem in the same way that Corollary 1 refines Theorem 2. The limiting system for (6) is

$$
X(t)=f_{0}-\int_{-\infty}^{t} a(t-s) g(X(s)) d s .
$$

Assumptions (B2) and (B3) insure that (7) has a unique constant solution $x_{0}$. Moreover, one can show that $x(t) \equiv x_{0}$ is the only bounded solution of (7). This proves

THEOREM 3. If (B1)-(B3) hold and if $x(t)$ is a bounded solution of (6), then $x(t) \rightarrow x_{0}$ as $t \rightarrow \infty$.

From Levin's results we see that if, in addition, $f^{\prime}(t)$ exists and is $L_{1}(0, \infty)$, then all solutions of (6) exist and are bounded for positive $t$. Other criterion can be given for boundedness. For example suppose $f(t)$ is bounded, $a(t)$ is $L_{1}(0, \infty)$ with $a(t) \geqq 0$ almost everywhere and $g(x)=\exp (x)-1$. If $x(t)$ is a solution of $(6)$, then for as long as it exists

$$
x(t) \geqq f(t)-\int_{0}^{t} a(t-s) d s>-M .
$$

Hence we also have

$$
x(t) \leqq f(t)+\int_{0}^{t} a(t-s) g(-M) d s<N .
$$

By general results of Nohel [3], $x(t)$ exists and is bounded on the interval $0 \leqq t<\infty$. 


\section{REFERENCES}

1. J. J. Levin, The qualitative behavior of a nonlinear Volterra equation, Proc. Amer. Math. Soc. 16 (1965), 711-718.

2. R. K. Miller, Asymptotic behavior of nonlinear delay differential equations, Journal of Differential Equations 1 (1965), 337-345.

3. J. A. Nohel, Some problems in nonlinear Volterra integral equations, Bull. Amer. Math. Soc. 68 (1962), 323-329.

4. R. E. A. C. Paley and N. Wiener, Fourier transforms in the complex domain, Amer. Math. Soc. Colloq. Publ. Vol. XIX, Amer. Math. Soc., Providence, R. I., 1934.

UNIVERSITY OF MiNNESOTA 